\title{
Femtosecond cubic optical nonlinearity of thin nickel films
}

\author{
P. J. Bennett, V. Albanis, Yu. P. Svirko, and N. I. Zheludev \\ Department of Physics and Astronomy, University of Southampton, Southampton SO17 1BJ, UK
}

Received July 23, 1999

\begin{abstract}
Transient pump-probe measurements of circular anisotropy in nickel films induced by 38-fs optical pulses show an instantaneous response that is related to the optical orientation of the spins of free electrons. Measurements in a sample of variable thickness, performed in both transmission and reflection, revealed that the surface significantly influences the degenerate cubic optical nonlinearity of the nickel films to a depth of approximately $4-5 \mathrm{~nm}$ into the bulk. (c) 1999 Optical Society of America

OCIS codes: $190.7110,190.4710,190.4350,160.3900,320.2250,320.7110$.
\end{abstract}

It was recently established that the cubic optical nonlinearity of metals associated with the optical orientation of spins is one of several mechanisms ${ }^{1-6}$ that play a very important role in the femtosecond optical response of bulk metals, ${ }^{7-9}$ in ferromagnetics in particular. ${ }^{10-13}$ The study of spin-related nonlinearity is of fundamental interest and practical importance, in particular for optical data storage. Here we report the results of a study of thin, semitransparent films of nickel of various thicknesses, using femtosecond timeresolved polarimetry. For what is believed to be the first time, we have combined transmission and reflection measurements and studied glass and air interfaces of nickel. We find that the ultrafast component of light-induced circular dichroism is insensitive to the optical anisotropy due to spontaneous magnetization of the film.

We identify the importance of the surface contribution to the film nonlinearity and also observe that not only the magnitude of the nonlinearity in nickel but also its sign can be different, depending on the nature of the interface. We also show that the nonlinearity is very fast and put an upper limit of $\sim 40 \mathrm{fs}$ on its relaxation time.

The nonlinearity of semitransparent nickel films of variable thickness was studied by use of an ultrafast polarization-sensitive pump-probe polarimeter with a Ti:sapphire laser with a central wavelength at $\lambda=$ $810 \mathrm{~nm}$, an average power at the sample of $\sim 50 \mathrm{~mW}$, a pulse duration of $\sim 38 \mathrm{fs}$, and a repetition rate of $82 \mathrm{MHz}$. A circularly polarized pump pulse induced circular birefringence and dichroism in the sample, which was then probed by a weaker linearly polarized pulse at the same wavelength. The probe propagated at a small angle to the pump, and both the probe and the pump were focused to a spot size of $130 \mu \mathrm{m}^{2}$. Using semitransparent nickel films deposited upon a glass substrate, we performed experiments in four different configurations, two reflective and two transmissive, from the air and glass substrate sides (see Figs. 1 and 2). In all cases the pump (dashed lines) was initially circularly polarized. The probe (solid lines) was initially linearly polarized, and its polarization azimuth was monitored after interaction with the sample. Our technique had an accuracy in measuring the pump-induced probe polarization-azimuth rotation of better than $10^{-6}$ rad and was based on continuous modulation of the probe polarization and the pump in- tensity. ${ }^{14}$ Unlike other techniques in which the circular birefringence-dichroism is calculated from the data on reflectivity for different polarizations, ${ }^{9}$ our technique is directly sensitive to the pump-induced circular birefringence-dichroism in the sample. This technique is practically insensitive to the background change of the sample reflectivity and the transmission. All these features make the technique particularly suitable for studying spin dynamics, as has been demonstrated in GaAs. ${ }^{14}$

Semitransparent nickel samples of variable thickness were deposited by electron-beam evaporation of 99.98\% pure nickel onto a glass substrate in a vacuum with a growth rate of approximately $1.5 \mathrm{~nm} / \mathrm{min}$. The substrates were glass microscope slides supplied by Chance Propper, Ltd. Measurements of the reflectivity at the air-nickel interface as a function of the transmission of the films (see Fig. 2) gave good agreement with the values calculated for pure nickel $[n=2.50+$ $i 4.41$ (Ref. 17)], indicating high quality and purity of the films. Our measurement of the natural polarization rotation of the sample owing to magneto-optical Kerr and Faraday effects (on reflection and in transmission) as a function of the incident polarization orientation showed spontaneous magnetization in the plane of the film and a slow variation of the magnetization direction along the sample.

The pump-induced polarization-azimuth rotation appeared on the background of the natural rotation owing to transverse spontaneous magnetization of the nickel sample. However, all our experiments showed that the pump-induced component of the rotation does not depend on the magnitude and the sign of the linear underlying (natural) polarization rotation. Within the available range of pump intensities, i.e., up to $16 \mathrm{GW} / \mathrm{cm}^{2}$, the rotation increased linearly with the pump intensity. We also observed that with the 38 -fs pump and probe pulses the nonlinear response was almost instantaneous (inset of Fig. 1). The rotation direction was reversed with opposite handedness of the pump, but its magnitude was unaffected. This observation is consistent with a picture of the pump inducing transient circular birefringence, which is a perturbation to the linear birefringence-dichroism induced by transverse spontaneous magnetization. The pump-induced probe-azimuth rotation when the pump was circularly polarized was at least a factor of 30 larger than the probe-azimuth rotation with the 

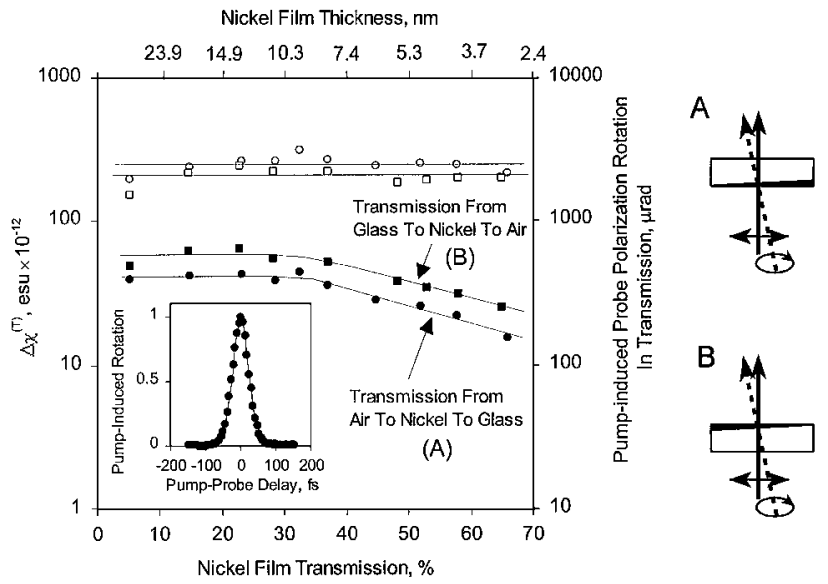

Fig. 1. Pump-induced probe polarization-azimuth rotation at zero pump-probe delay observed in transmission experiments for opposite directions of propagation ( and a) and the corresponding values of the optical nonlinearity $\Delta \chi^{(T)}$ in nickel, calculated from Eq. (1) ( $\bigcirc$ and $\left.\square\right)$. The inset shows the normalized pump-induced probe polarization azimuth rotation as a function of the pump-probe delay in transmission through the sample. In all cases the response was instantaneous within the time resolution available, with 38-fs optical pulses.

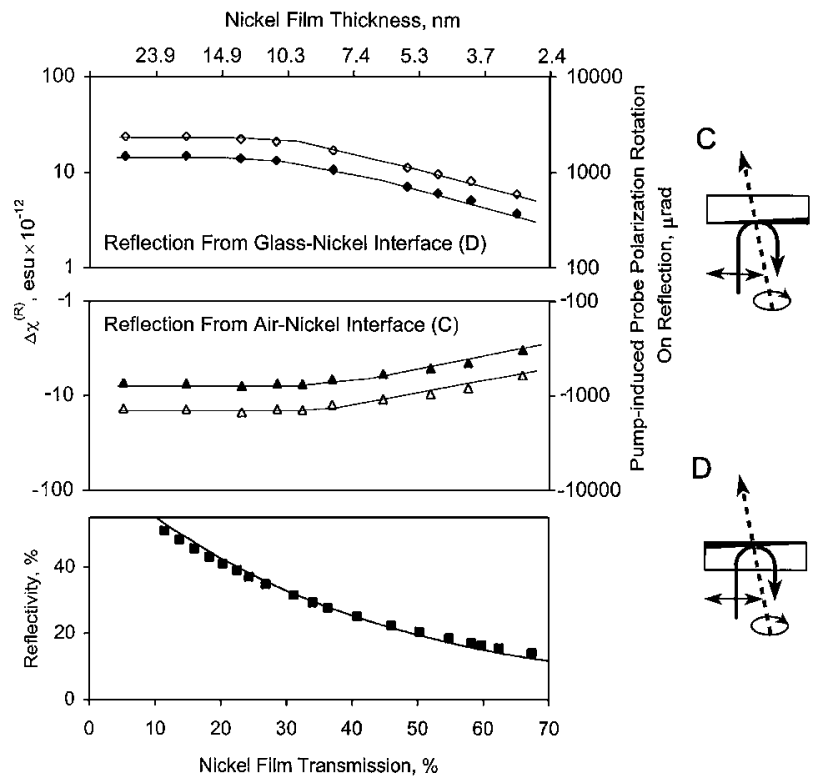

Fig. 2. Pump-induced probe polarization-azimuth rotation at zero pump-probe delay on reflection as a function of the transmission of the nickel film. Note that the direction of the rotation at the nickel-air interface $(\boldsymbol{\Delta})$ is the opposite of that at the glass-air interface $(\bullet)$. The lefthand scale shows the corresponding effective nonlinearity $\Delta \chi^{(R)}$, as calculated from Eq. (2) $(\triangle$ and $\diamond)$. The bottom graph shows a plot of the reflectivity from the air-nickel interface (ם). The solid curve is calculated from a numerical model for a refractive index of $n=2.50-i 4.41 .^{15}$

pump linearly polarized at $45^{\circ}$ to the probe. For the results reported below, the pump was always circularly polarized.

In transmission experiments we found that the sign of the pump-induced probe polarization is independent of the direction of propagation through the sample and depends only on the handedness of the pump pulse. For a given pump intensity, the rotation tends to increase with the sample thickness and is slightly higher for propagation from the glass substrate into the nickel film. Such a difference in rotation may be anticipated, as the reflectivity of the two interfaces, and therefore the intensities inside the nickel film, are different. Presuming that the film is homogeneous, we can calculate the nonlinearity of the nickel layer from the following formula ${ }^{16}$ :

$$
\Theta^{T}= \pm \frac{32 \pi^{3}}{c \lambda\left|1+\frac{n}{n_{0}}\right|^{2}} \operatorname{Re}\left\{\frac{\chi_{x x y y}-\chi_{x y y x}}{n}\right\} \int_{0}^{L} I_{p}(z) \mathrm{d} z
$$

Here $\Theta^{T}$ is the pump-induced probe polarizationazimuth rotation in transmission through a sample of length $L$ and $I_{p}(z)$ is the intensity of the pump wave in the sample. Equation (1) is valid when the optical response is instantaneous, as was observed in our experiments. Equation (1) also assumes a weak dependence of the nonlinear susceptibilities and the complex refractive index on frequency within the laser pulse spectrum, which is the case in metals. Equation (1) neglects multiple reflections, which is a reasonable assumption for a highly absorbing sample. This formula is suitable for propagation in both directions; however, if the light enters the nickel film from the glass side, $n_{0}=1.50$ should be assumed, but for the opposite direction one has to use refractive index $n_{0}=1$. The plus and minus signs refer to right- and left-hand circular polarizations of the pump, respectively. Here the nonlinearity has been introduced in standard fashion by use of the nonlinear constitutive equation, $P_{i}^{\mathrm{nl}}(\omega)=\chi_{i j k l}(\omega, \omega, \omega,-\omega) E_{j}(\omega) E_{k}(\omega) E_{l}{ }^{*}(\omega)$. The coherent contribution to the nonlinear rotation, which depends on the mutual phase between the pump and the probe, was neglected in Eq. (1), as in our experiments the coherent component was suppressed by use of the technique described in Ref. 17.

The effective nonlinearity that was recovered from the transmission experiment is presented in Fig. 1, in which the following notation is used: $\Delta \chi^{(T)}=\operatorname{Re}$ $\times\left[\left(\chi_{x x y y}-\chi_{x y y x}\right) / n\right]$. One can see that the nonlinearity is practically independent of the film thickness, $\Delta \chi^{(T)} \sim 2 \times 10^{-10}$ esu. Neglecting multiple reflections and modeling the pump intensity as a decaying exponential across the film lead to an error of less than $15 \%$ in the estimate of the nonlinearity. A small difference between the values of the nonlinearities derived from the data for the opposite directions of preparations can be attributed to a surface contribution that we discuss below. Our transmission measurements suggest that the nonlinearity, $\Delta \chi^{(T)}=\operatorname{Re}\left[\left(\chi_{x x y y}-\chi_{x y y x}\right) / n\right]$, in nickel can be approximated to be homogeneous within the range of thicknesses measured in our experiment, with the surface effects making only a small contribution.

The results of our measurements of the reflected light suggest a very different picture for the nonlinearity that is responsible for the reflective effects: $\Delta \chi^{(R)}=\operatorname{Im}\left(\left(\chi_{x x y y}-\chi_{x y y x}\right) /\left\{n\left[1-\left(n / n_{0}\right)^{2}\right]\right\}\right)$. For a 
homogeneous nickel layer it would be expected that the induced rotation sign would be independent of which interface the light is reflected from. However, this expectation was not confirmed experimentally (see Fig. 2).

To our astonishment we observed the opposite sign of rotation when the sample was turned over and illuminated from the opposite side, for the same handedness of the pump polarization with the same experimental setup. We emphasize again that the pump-induced polarization rotation is independent of the spontaneous magnetization. If we again assume that the film was homogeneous and calculate its nonlinearity by use of a formula derived with this assumption, ${ }^{16}$

$$
\Theta^{R}= \pm \frac{32 \pi^{2} I_{p}}{c n_{0}\left|1+\frac{n}{n_{0}}\right|^{2}} \operatorname{Im}\left\{\frac{\chi_{x x y y}-\chi_{x y y x}}{n\left[1-\left(\frac{n}{n_{0}}\right)^{2}\right]}\right\}
$$

we find that the nonlinearity on opposite sides of the film will have different signs (see Fig. 2). This result shows that in nickel films the nonlinearity, $\Delta \chi^{(R)}=$ $\operatorname{Im}\left(\left(\chi_{x x y y}-\chi_{x y y x}\right) /\left\{n\left[1-\left(n / n_{0}\right)^{2}\right]\right\}\right)$, is very inhomogeneous and that the surface strongly affects the optical properties of the medium. It is possible to estimate the depths, $\xi_{1}$ and $\xi_{2}$, to which the two surfaces influence the optical response of the material from our measurements. As nonlinearities at different interfaces have different signs, if $\xi_{1} \approx \xi_{2}=\xi$, the film thickness at which the nonlinearity calculated from Eq. (2) starts to decrease is approximately $2 \xi$, when the layers with opposite signs of nonlinearity start to overlap, giving $\xi \approx 4-5 \mathrm{~nm}$.

We believe that the bulk optical nonlinearity in nickel films has the same origin as the ultrafast component of the polarization-sensitive optical response that was recently observed in $\mathrm{CoPt}_{3}$ alloy films. ${ }^{9}$ The nonlinearity is related to the spin polarization of electrons excited with a circularly polarized pump. This nonlinearity was first discussed with relevance to metals in Ref. 7. Following the impulse of photoexcitation with a circularly polarized pump, an electron-spin distribution is created with finite net spin polarization, giving rise to circular birefringence-dichroism in the sample. The net spin polarization decays through electron-electron scattering processes. As the induced birefringence-dichroism is instantaneous within the pulse duration, this puts an upper limit on the spin relaxation time of $\approx 40 \mathrm{fs}$. As the electroncollision time in nickel is $\sim 2 \mathrm{fs}$, our results show that the spin-flipping occurs on average at least every 20 collisions. The spin relaxation time can be compared with the electron-spin relaxation time that is due to exchange interaction and spin-orbit coupling, which was recently calculated to be a few femtoseconds for nickel. ${ }^{18}$ The spin relaxation time, which governs the observed nonlinearity response time, should be clearly distinguished from the much longer electron-thermalization time, which was measured to be $280 \pm 30 \mathrm{fs}$ in nickel, ${ }^{13}$ and also from the evenlonger spin-lattice relaxation time, which is a few hundred picoseconds for nickel..$^{18,19}$

The dramatic change of the nonlinearity at the interface from the bulk observed in our experiments, and in particular the change of the sign of the pumpinduced rotation on reflection from the nickel-air and the nickel-glass interfaces, is not completely understood. It may be related to specific surface states created by the presence of the interface or to interface contamination-oxidation, or it may be related to the local field effects owing to surface roughness. It is also known that the spin relaxation processes at the interface are considerably different from those in the bulk. ${ }^{20}$

The authors thank B. D. Rainford and V. V. Shuvalov for their discussions of this work and Goodfellow Cambridge, Ltd., and NATO (grant CRGP960274) for their financial support. N. I. Zheludev's e-mail address is n.i.zheludev@soton.ac.uk.

\section{References}

1. W. K. Burns and N. Bloembergen, Phys. Rev. B 4, 3437 (1971).

2. G. L. Eesley, Phys. Rev. B 33, 2144 (1986).

3. R. W. Schoenlien, W. Z. Lin, J. G. Fujimoto, and G. L. Eesley, Phys. Rev. Lett. 58, 1680 (1987).

4. X. Y. Wang and M. C. Downer, Opt. Lett. 17, 1450 (1992).

5. C. K. Sun, F. Vallee, L. H. Acioli, E. P. Ippen, and J. G. Fujimoto, Phys. Rev. B 50, 15337 (1994).

6. A. Liebsch, Surf. Sci. 307/309, 1007 (1994).

7. N. I. Zheludev, P. J. Bennett, H. Loh, S. V. Popov, I. R. Shatwell, Yu. P. Svirko, V. E. Gusev, V. F. Kamalov, and E. V. Slobodchikov, Opt. Lett. 20, 1368 (1995).

8. S. Dhanjal, S. V. Popov, I. R. Shatwell, Yu. P. Svirko, N. I. Zheludev, and V. E. Gusev, Opt. Lett. 22, 1879 (1997).

9. G. P. Ju, A. Vertikov, A. V. Nurmikko, C. Canady, G. Xiao, R. F. C. Farrow, and A. Cebollada, Phys. Rev. B 57, R700 (1998).

10. R. P. Pan, H. D. Wei, and Y. R. Shen, Phys. Rev. B 39, 1229 (1989).

11. T. Rasing, M. G. Koerkamp, B. Koopmans, and H. Vander Berg, J. Appl. Phys. 79, 6181 (1996).

12. E. Beaurepaire, J. C. Merle, A. Daunois, and J. Y. Bigot, Phys. Rev. Lett. 76, 4250 (1996).

13. J. Hohlfeld, E. Matthias, R. Knorren, and K. H. Benneman, Phys. Rev. Lett. 78, 4861 (1997).

14. A. R. Bungay, S. V. Popov, I. R. Shatwell, and N. I. Zheludev, Phys. Lett. A 234, 379 (1997).

15. E. D. Palik, ed., Handbook of Optical Constants of Solids (Academic, London, 1985), p. 323.

16. Yu. P. Svirko and N. I. Zheludev, Polarization of Light in Nonlinear Optics (Wiley, New York, 1998), Chap. 7.

17. S. V. Popov, N. I. Zheludev, and Yu. P. Svirko, Opt. Lett. 19, 13 (1994).

18. W. Hubner and G. P. Zhang, Phys. Rev. B 58, R5920 (1998).

19. A. Scholl, L. Baumgarten, R. Jacquemin, and W. Eberhardt, Phys. Rev. Lett. 79, 5146 (1997).

20. V. F. Gantmaher and I. B. Levinson, Scattering in Metals and Semiconductors (Nauka, Moscow, 1984), Chap. 13. 